\title{
ANALYSIS OF HYSTERETIC REACTION-DIFFUSION SYSTEMS
}

\author{
BY \\ CHICHIA CHIU (Department of Mathematics, Michigan State University, East Lansing, MI)
}

AND

NOEL WALKINGTON (Department of Mathematics, Carnegie Mellon University, Pittsburgh, PA)

\begin{abstract}
In this paper, we consider a mathematical model motivated by patterned growth of bacteria. The model is a system of differential equations that consists of two sub-systems. One is a system of ordinary differential equations and the other one is a reaction-diffusion system. Pattern formation in this model is caused by an initial instability of the ordinary differential equations. However, nonlinear coupling to the reaction-diffusion system stabilizes the ordinary differential equations resulting in stationary long-time behavior. We establish existence, uniqueness, and characterize long-time behavior of the solutions.
\end{abstract}

1. Introduction. It has frequently been observed in biological experiments that an initially uniform distribution of cells may grow and develop an intricate distribution when the nutrient concentration is non-uniform. This phenomenon is referred to as patterned cell growth. Below we analyze a system of equations that has been proposed to model such systems. Our results show that solutions of the equations will reflect some of the behavior observed in the experiments.

Various models have been proposed to explain this pattern formation. In [6] and [7], it is assumed that the growth rate of cells has a hysteresis dependence upon nutrient and buffer, and this leads to a model exhibiting such patterns. Budriené, Polezhaev, and Ptitsyn [2] developed a more refined model that recognizes that the cells may be in one of two states, active and inactive. ${ }^{1}$ The transition between the two depends on

Received February 14, 1995.

1991 Mathematics Subject Classification. Primary 35K57, 65N12, 92-08, 92B05.

Key words and phrases. Reaction-diffusion equations, accretion pattern formation, semi-groups.

This work was supported in part by NSF Grant No. DMS-9206677. The research was carried out when the first author was visiting the Department of Mathematics and the Center for Nonlinear Analysis at Carnegie Mellon University.

The second author was supported by National Science Foundation Grant No. DMS-9203406 while this work was undertaken. This work was also supported by the Army Research Office and the National Science Foundation through the Center for Nonlinear Analysis.

E-mail address: chiu@math.msu.edu

E-mail address: nwOy@cmu.edu

${ }^{1}$ We use the terms active and inactive throughout this paper. In [2] active cells were referred to as vegetative, and inactive as anabiotic. 
the total cell population, disintegration rate (assumed constant) and diffusion rate of the mediator. The model we study here was first proposed in $[4,7,2,6]$ and involves a system of reaction-diffusion and ordinary differential equations. The diffusion equations model the motion of various chemicals (nutrients, etc.) and ordinary differential equations model the growth of active and inactive cells. In this model the ordinary differential equations are unstable at the uniform state; however, as the solution evolves, nonlinear effects modify the coefficients of these equations so that they become stable, and steadystate patterns are formed for all the quantities involved. This mechanism of pattern formation is referred to as accretion. Hysteresis enters the problem due to the fact that in certain environments a cell may be either active or inactive depending upon the history. Some related theories and an extensive bibliography on hysteresis are given in the survey by Macki et al. [16]. More recently, Little and Showalter [14] and Hornung and Showalter $[9,10]$ have incorporated hysteresis into the framework of maximal monotone (m-accretive) operators, obtaining existence and well-posedness for problems involving phase changes.

In the next section we introduce such systems of reaction-diffusion equations and recall their essential structure. A system consisting of two diffusion equations, modeling the motion of nutrient and buffer, and two ordinary differential equations, modeling active and inactive cells, is introduced, and a numerical calculation is presented to illustrate the behavior that solutions of these equations may exhibit. In Sec. 3, we establish existence and uniqueness of the solutions for this problem using semigroup theory. Section 4 characterizes the large-time asymptotic behavior of the solutions. The large-time asymptotics show ultimate stability of the system, and verify that a steady-state pattern of inactive cells will form.

\section{Hysteretic reaction-diffusion systems.}

2.1. General equations. In this section, we give a general formulation of hysteretic reaction-diffusion systems and then specialize them to a system with four unknown functions, which is subsequently analyzed. Letting $\Omega \subset R^{2}$ be a bounded, connected 2-dimensional region with a smooth boundary $\partial \Omega$, the hysteretic reaction-diffusion systems take the form:

$$
\left\{\begin{array}{l}
\mathbf{u}_{t}+A \mathbf{u}=F_{1} \mathbf{u} \\
\mathbf{h}_{t}-\operatorname{div}(D \nabla \mathbf{h})=-F_{2} \mathbf{u}
\end{array} \quad x \in \Omega, t \in[0, \infty)\right.
$$

where

- $\mathbf{u}=\mathbf{u}(x, t)=\left(u_{1}, \ldots, u_{\mu}\right)^{\mathrm{T}}$ is a $\mu$-dimensional vector function;

- $\mathbf{h}=\mathbf{h}(x, t)=\left(h_{1}, \ldots, h_{\nu}\right)^{\mathrm{T}}$ is a $\nu$-dimensional vector function;

- $A=A(x, t, \mathbf{h})=\left(a_{i j}\right)_{1 \leq i, j \leq \mu}$ is a $\mu \times \mu$ matrix function;

- $F_{1}=F_{1}(x, t, \mathbf{h})=\left(f_{1 i j}\right)_{1 \leq i, j \leq \mu}$ is a $\mu \times \mu$ nonnegative matrix function, i.e., $f_{1 i j} \geq 0$ for all $i j$;

- $F_{2}=F_{2}(x, t, \mathbf{h})=\left(f_{2 i j}\right)_{1 \leq i \leq \nu, 1 \leq j \leq \mu}$ is a $\nu \times \mu$ nonnegative matrix function, i.e., $f_{2 i j} \geq 0$ for all $i j$;

- $D=D(x, t, \mathbf{h})$ is a positive-definite fourth-order tensor. 
For cell biology, $\mathbf{u}$ is the cell density vector which might represent different levels of torpor; $\mathbf{h}$ represents different substrate resources that are related to cell growth; $A \mathbf{u}$ simulates the transition process between different torpor levels; $F_{1} \mathbf{u}$ simulates the growth of cells by uptake of the nutrient $(\mathbf{h}) ;-F_{2} \mathbf{u}$ simulates the degradation of substrate through uptake of nutrient by actively growing cells; and $\operatorname{div}(D \nabla \mathbf{h})$ is the diffusion process of substrate chemicals. We assume that cells are non-mobile, so that the first part of (1) is a system of ordinary differential equations and the second part is a reaction-diffusion system. ${ }^{2}$ The system (1) is said to be hysteretic because the matrix $A$ is usually defined in such a way that transition between levels of torpor has a hysteretic dependence on the availability of substrate concentrations. In this paper, we study the case that substrate chemicals are provided initially and there is no further supply during the process of pattern formation. The initial and boundary conditions then become:

$$
\left\{\begin{array}{l}
\mathbf{u}(x, 0)=\mathbf{u}_{0}(x) \quad \text { and } \quad \mathbf{h}(x, 0)=\mathbf{h}_{0}(x) \quad \text { for } x \in \Omega \\
\left.(D \nabla \mathbf{h}) n\right|_{\partial \Omega}=0
\end{array}\right.
$$

where $n$ is the outward normal direction on $\partial \Omega$.

2.2. Conservation property. We assume that there is no loss or production of cells during transition between levels of torpor, and that cells can only disappear through cell fission. These assumptions lead to the following conservation hypotheses.

(i) $\sum_{i=1}^{\mu} a_{i j}=0$, for every $j$,

(ii) There exist constants $\mathbf{c}=\left(c_{1}, c_{2}, \ldots, c_{\nu}\right)$ such that

$$
\sum_{i=1}^{\mu} \sum_{j=1}^{\mu} f_{1 i j} u_{j}-\sum_{i=1}^{\nu} \sum_{j=1}^{\mu} c_{i} f_{2 i j} u_{j}=0
$$

To see that this results in a conserved property, multiply both sides of the equation for $h_{k}$ by $c_{k}$ and sum over all of the equations of the system to get

$$
\sum_{j=1}^{\mu} \frac{\partial u_{j}}{\partial t}+\sum_{k=1}^{\nu} c_{k} \frac{\partial h_{k}}{\partial t}=\operatorname{div}(\mathbf{c} D \nabla \mathbf{h})
$$

Integrating this over $\Omega$ and using the divergence theorem with the zero flux boundary condition gives

$$
\frac{\partial}{\partial t}\left[\sum_{j=1}^{\mu} \int_{\Omega} u_{j} d x+\sum_{k=1}^{\nu} c_{k} \int_{\Omega} h_{k} d x\right]=0
$$

\footnotetext{
${ }^{2}$ It is possible to consider mobile types of cells, so that the whole system is of reaction-diffusion type. Another possibility is to use a reaction-diffusion-chemotaxis model, i.e., cells tend to move towards sources of nutrients.
} 
It follows that

$$
\int_{\Omega} \sum_{j=1}^{\mu} u_{j}+\sum_{k=1}^{\nu} c_{k} h_{k}=C
$$

where $C$ is a constant.

2.3. A two-component system. In this paper, we consider a simple case of system (1), which involves only two levels of torpor, active and inactive, and two substrate concentrations, nutrient and buffer. Let $u$ and $v$ be the active and the inactive cell concentrations respectively, $H$ be the nutrient concentration, and $G$ be the buffer concentration. Then the system is

$$
\left\{\begin{array}{l}
\frac{\partial}{\partial t}\left(\begin{array}{l}
u \\
v
\end{array}\right)+\left(\begin{array}{cc}
a & -b \\
-a & b
\end{array}\right)\left(\begin{array}{l}
u \\
v
\end{array}\right)=\left(\begin{array}{c}
f u \\
0
\end{array}\right), \\
\frac{\partial}{\partial t}\left(\begin{array}{l}
H \\
G
\end{array}\right)-\left(\begin{array}{cc}
D_{H} & 0 \\
0 & D_{G}
\end{array}\right)\left(\begin{array}{l}
\Delta H \\
\Delta G
\end{array}\right)=\left(\begin{array}{c}
-f u / Y_{H} \\
-f u / Y_{G}
\end{array}\right) .
\end{array}\right.
$$

Here, $f=f(G, H), a=a(G, H)$, and $b=b(G, H)$ are three nonnegative functions depending on the product $G H$ which is the combined concentration of nutrient and buffer. When $b>0$, environmental conditions favor growth and inactive cells may become active. On the other hand, if $a>0$, then the conditions are less favorable and active cells switch to the inactive state. The function $f$ represents growth of cells by cell fission. $D_{H}>0$ and $D_{G}>0$ are diffusion constants. The two terms on the righthand side of the second system describe loss of $H$ and $G$ due to uptake by active cells. $Y_{H}>0$ and $Y_{G}>0$ are two-cell-yield constant parameters. Note that if $a=0$ and $b$ and $f$ are positive, the ordinary differential equation will cause $u$ to grow exponentially. Typically the initial conditions will cause this to occur for $t$ small. However, as $u$ grows, the diffusion equations cause $G$ and $H$ to be reduced (the active cells consume these quantities). As $G$ and $H$ reduce, this will alter the coefficients $a$ and $b$. Typically the evolution will yield $b=0$ and $0<f<a$, causing $u$ to decay so that a steady state is attained.

We assume that all cells at the start are active and that the distributions of $u, H$, and $G$ at $t=0$ are known. The initial conditions are then

$$
\begin{gathered}
u(x, 0)=u_{0}(x) \geq 0 \quad \text { and } \quad v(x, 0)=0, \\
H(x, 0)=H_{0}(x) \quad \text { and } \quad G(x, 0)=G_{0}(x) .
\end{gathered}
$$

The boundary conditions for $H$ and $G$ are

$$
\left.\frac{\partial H}{\partial n}\right|_{\partial \Omega}=\left.\frac{\partial G}{\partial n}\right|_{\partial \Omega}=0 .
$$

The parameter functions $f, a$, and $b$ are determined by the following criteria:

1. $f(\cdot)$ is monotone increasing and bounded, $f(0)=0$.

2. When $G H$ is large (acceptable environmental condition), $a=0$ and $b>0$.

3. When $G H$ is small (unacceptable environmental condition), $a>0$ and $b=0$.

4. $a(\cdot)$ is monotone decreasing or zero; $b(\cdot)$ is monotone increasing or zero and

$$
b_{\max }=\sup _{\alpha \geq 0} b(\alpha)=\lim _{\alpha \rightarrow \infty} b(\alpha)>0 .
$$




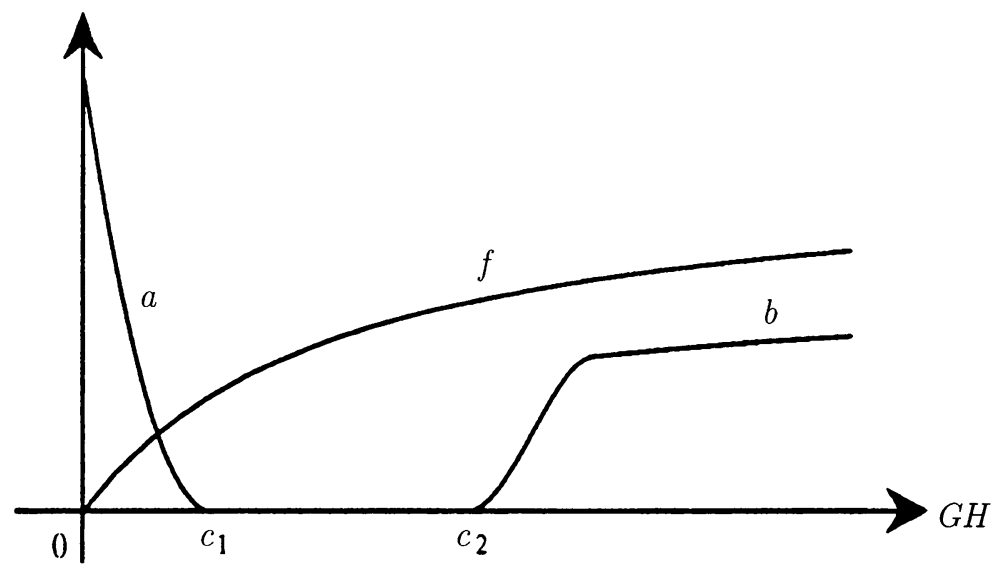

FIG. 1. Graph of parameter functions: $f(G H), a(G H)$, and $b(G H)$

5. There is a gap $\left[c_{1}, c_{2}\right], c_{1} \leq c_{2}$, such that

$$
a(\alpha)=b(\alpha)=0 \text { for all } \alpha \in\left[c_{1}, c_{2}\right] ;
$$

so active cells and inactive cells do not switch to each other when $G H \in\left[c_{1}, c_{2}\right]$. This property is exhibited in experiments; thus cells can have a hysteresis in growth depending on the combined concentration of nutrient and buffer [6].

An illustrative graph of $f, a$, and $b$ is given in Fig. 1. Although this system is a special case of (1), we show subsequently that it contains all of the essential features to model accretion.

2.4. A numerical example. Our analysis of the two-component system is rather technical, but the outcome is pleasantly simple. Under reasonable assumptions on the data, the solution of (3) tends to a steady state where the buffer and nutrient concentrations, $G$ and $H$, are constant, the concentration of active cells, $u$, tends to zero, and the only nontrivial part of the steady-state solution is the distribution of inactive cells, $v$. We demonstrate this with a numerical solution that illustrates concentric ring patterns that often form in bacterial growth when a drop of nutrient is placed at the center of an agar pour plate (e.g., Hauser, 1885, [8]).

We choose the growth rate function $f(G H)$ according to Monod's law [17], [19], and let $a$ and $b$ also depend upon $G$ and $H$ through the product $G H$ as illustrated in Fig. 1. The diffusion coefficients and yield parameters were set to $D_{H}=0.025, D_{G}=0.005$, $Y_{H}=10$, and $Y_{G}=1$. Since our numerical solution did converge to a steady state with $G$ and $H$ constant and $u$ zero, we only show inactive cell densities at several times in Fig. 2 (see p. 94). The concentric pattern observed in this figure resembles the patterns observed in the biological experiments reported in [6] and [8].

The analysis presented below was partially motivated by the desire to construct efficient numerical schemes that would approximate the biological equations. For example, the large-time asymptotics established in Sec. 4 follow from the conservation property discussed above and nonnegativity of the solution. In [5] the authors construct a nu- 

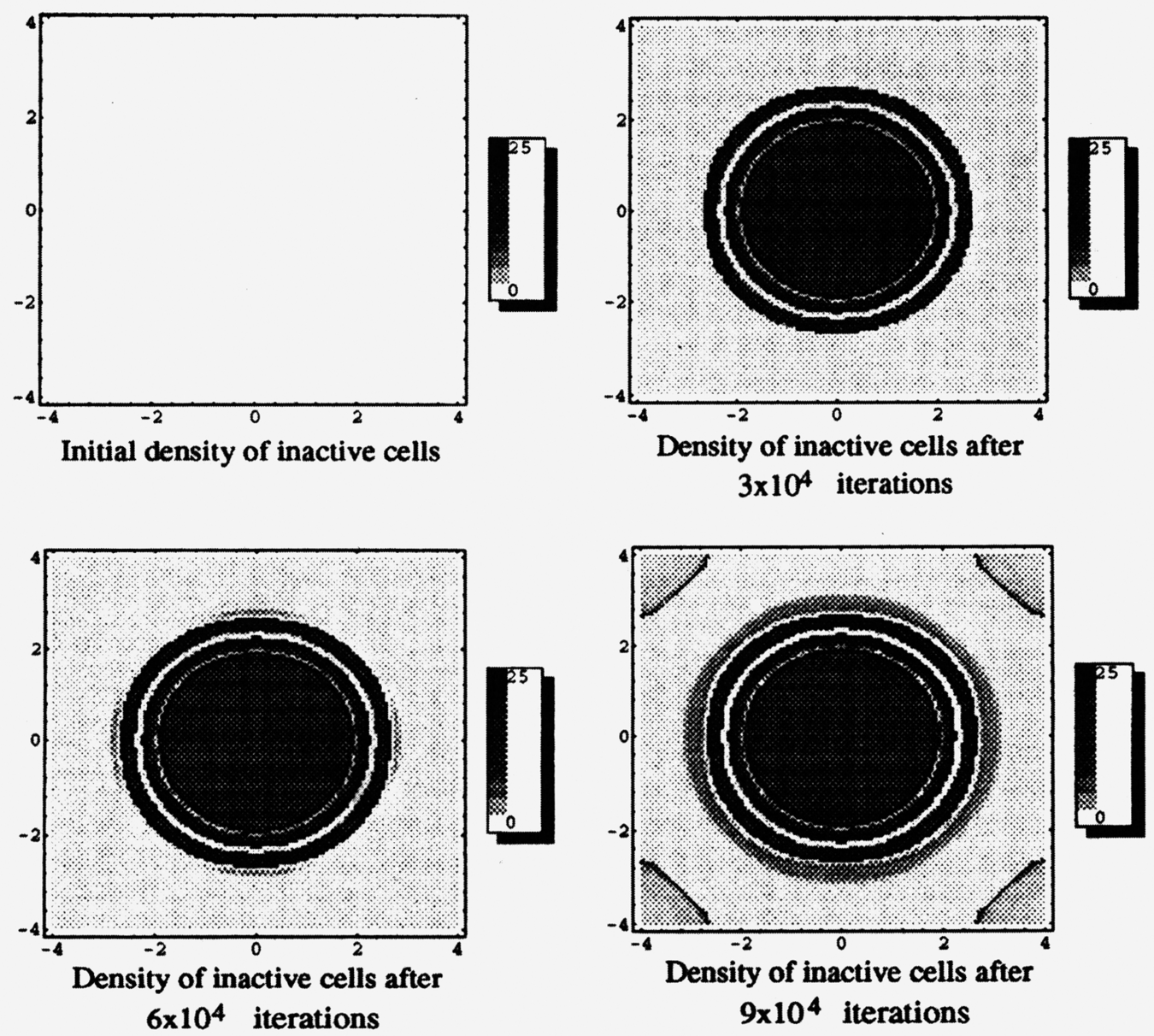

FIG. 2. Density plots of inactive cells

merical scheme that satisfies discrete versions of these properties and characterize the large-time asymptotics for the numerical example exhibited here.

\section{Existence of solutions.}

3.1. Abstract semilinear equations. To establish existence of a unique solution to (3) we collect some standard results from the theory of semigroups and semilinear equations. The following theorem from [3] establishes existence of mild solutions to semilinear equations when the principle part generates a $C^{0}$ semigroup.

Theorem 3.1. Let $-\mathcal{A}$ be the generator of a $C^{0}$ semigroup $\mathcal{S}$ on the Banach space $\mathcal{X}$ and let $g: \mathfrak{R}^{+} \times \mathcal{X} \rightarrow \mathfrak{R}$ be continuous and satisfy the Lipschitz condition: $\forall t>0$, there exists $K(t)>0$ such that for $x, y \in \mathcal{X}$,

$$
\|g(s, x)-g(s, y)\|_{\mathcal{X}} \leq K(t)\|x-y\|_{\mathcal{X}}, \quad 0 \leq s \leq t .
$$


Then for each $u_{0} \in \mathcal{X}$ there is a unique mild solution $u \in C[0, \infty ; \mathcal{X})$ of

$$
\frac{d u}{d t}+\mathcal{A} u=g(t, u), \quad u(0)=u_{0},
$$

i.e., $u$ satisfies the integral equation

$$
u(t)=\mathcal{S}(t) u_{0}+\int_{0}^{1} \mathcal{S}(t-s) g(s, u(s)) d s .
$$

Remarks. 1) This result is established using the Banach fixed point theorem to determine a fixed point of the integral equation above.

2) The continuity of $g$ with respect to its first variable is used to verify that the map $[0, \infty) \rightarrow g(\cdot, u(\cdot))$ is strongly measurable for each choice of $u \in C[0, \infty ; \mathcal{X})$. For specific examples, this assumption may be relaxed. For example, if $\mathcal{X}=L^{p}(\Omega), 1 \leq p<\infty$, and $g(s, u)=\psi(s) u$ where $\psi(s) \in L^{\infty}(\Omega)$ is given by $\psi(s)(x)=\Psi(s, x)$ with $\Psi \in$ $L^{\infty}((0, \infty) \times \Omega)$, then $g(\cdot, u(\cdot))$ is clearly measurable for any choice of $u \in C[0, \infty ; \mathcal{X})$.

3 ) The proof of the above theorem extends in a routine manner to the slightly more general situation where $g: C[0, \infty ; \mathcal{X}) \rightarrow C[0, \infty ; \mathcal{X})$ with corresponding hypothesis

$$
\|g(x)-g(y)\|_{C[0, t ; \mathcal{X}]} \leq K(t)\|x-y\|_{C[0, t ; \mathcal{X}]}, \quad \forall t>0 .
$$

4) If $-\mathcal{A}$ generates an analytic semigroup, then a sufficient condition for $u$ to be a strong solution (i.e., $u \in C^{1}(0, \infty ; \mathcal{X})$ and $\left.u(t) \in D(\mathcal{A})\right)$ is that $u_{0} \in D(\mathcal{A})$ and $g(u)(\cdot)$ be Hölder continuous.

The next lemma states an additional Lipschitz condition that will guarantee a strong solution when the principle part of the equation generates an analytic semigroup.

LEMma 3.2. Let $-\mathcal{A}$ be the generator of an analytic semigroup $\mathcal{S}$ on the Banach space $\mathcal{X}$, and suppose that $g: C[0, \infty ; \mathcal{X}) \rightarrow C[0, \infty ; \mathcal{X})$ satisfies the Lipschitz condition: $\forall t>0$, there exists $K(t)>0$ such that for $x \in C[0, \infty ; \mathcal{X}), \delta>0$,

$$
\|g(x)(s+\delta)-g(x)(s)\|_{\mathcal{X}} \leq K(t)\left(\delta+\|x(s+\delta)-x(s)\|_{\mathcal{X}}+\int_{0}^{s}\|x(\xi+\delta)-x(\xi)\|_{\mathcal{X}} d \xi\right),
$$

$0 \leq s \leq t$. If $u_{0} \in D(\mathcal{A})$, then a mild solution of $d u / d t+\mathcal{A} u=g(u), u(0)=u_{0}$, is also a strong solution.

Proof. Fix $T>0$. We will show that $u$ is Lipschitz on $[0, T]$; the Lipschitz hypothesis will then imply that $g(u)$ is Lipschitz on $[0, T]$. If $u \in C[0, \infty ; \mathcal{X})$ is a mild solution with $u(0)=u_{0}$, taking the difference of the integral expression for $u(t+\delta)$ and $u(t)$ for $0 \leq t \leq T$ gives

$$
\begin{aligned}
u(t+\delta)-u(t) & =[\mathcal{S}(t+\delta)-\mathcal{S}(t)] u_{0} \\
& +\int_{t}^{t+\delta} \mathcal{S}(s) g(u)(t+\delta-s) d s+\int_{0}^{t} \mathcal{S}(s)[g(u)(t+\delta-s)-g(u)(t-s)] d s .
\end{aligned}
$$


Recall that if $u_{0} \in D(\mathcal{A})$ then $\mathcal{S}(\cdot) u_{0}$ is Lipschitz with constant $\left\|\mathcal{A} u_{0}\right\|_{\mathcal{X}}$. Also, since $g(u) \in C[0, \infty ; \mathcal{X})$ it follows that the second term on the right may be bounded by $C(T) \delta$. The last term is bounded using the Lipschitz hypothesis:

$$
\begin{aligned}
& \left\|\int_{0}^{t} \mathcal{S}(s)[g(u)(t+\delta-s)-g(u)(t-s)] d s\right\|_{\mathcal{X}} \\
& \quad \leq C(T) \int_{0}^{t}\|g(u)(\delta+s)-g(u)(s)\|_{\mathcal{X}} d s \\
& \quad \leq K(T) \int_{0}^{t}\left(\delta+\|u(\delta+s)-u(s)\|_{\mathcal{X}}+\int_{0}^{s}\|u(\xi+\delta)-u(\xi)\|_{\mathcal{X}} d \xi\right) d s \\
& \quad \leq K(T) T \delta+K(T)(1+T) \int_{0}^{t}\|u(\delta+s)-u(s)\|_{\mathcal{X}} d s .
\end{aligned}
$$

Combining the estimates gives for $0 \leq t \leq T$

$$
\|u(t+\delta)-u(t)\|_{\mathcal{X}} \leq C\left(u_{0}, T\right) \delta+C(T) \int_{0}^{t}\|u(s+\delta)-u(s)\|_{\mathcal{X}} d s .
$$

Gronwall's inequality now yields

$$
\|u(t+\delta)-u(t)\|_{\mathcal{X}} \leq C\left(u_{0}, T\right) e^{C(T) t} \delta, \quad 0 \leq t \leq T,
$$

so that $u$ is Lipschitz (and hence Hölder) continuous on bounded subsets of $\mathfrak{R}^{+}$. The Lipschitz hypothesis now implies $g(u)$ is also Lipschitz continuous on bounded subsets and in this situation mild solutions are strong [3].

REMARK. It suffices to consider $u_{0} \in D\left(\mathcal{A}^{\alpha}\right)$ for some $\alpha>0$, since $u_{0} \in \mathcal{X}=D\left(\mathcal{A}^{0}\right)$ implies $\mathcal{S}(\cdot) u_{0} \in C[0, T ; \mathcal{X}]$ and $u_{0} \in D(\mathcal{A})$ implies $\mathcal{S}(\cdot) u_{0} \in C^{1}[0, T ; \mathcal{X}]$; so by interpolation $\mathcal{S}(\cdot) u_{0}$ will be Hölder continuous on the interpolated spaces $D\left(\mathcal{A}^{\alpha}\right)$.

We finally state an elementary identity that will be required later. This identity can be motivated by considering the evolution equation satisfied by $\exp (-\lambda t) u(t)$.

Lemma 3.3. Let $\mathcal{S}$ be a $C^{0}$ semigroup on $\mathcal{X}, g \in C[0, \infty ; \mathcal{X})$, and $u \in C[0, \infty ; \mathcal{X})$ be defined by the variation of parameters formula

$$
u(s)=\mathcal{S}(s) u_{0}+\int_{0}^{s} \mathcal{S}(t-\xi) g(\xi) d \xi
$$

Then

$$
u(t)=e^{-\lambda t} \mathcal{S}(t) u_{0}+\int_{0}^{t} e^{-\lambda(t-s)} \mathcal{S}(t-s)(\lambda u(s)+g(s)) d s .
$$

Proof. We "multiply" the equation for $u(s)$ by $\lambda e^{-\lambda(t-s)} \mathcal{S}(t-s)$ and integrate from zero to $t$. Upon changing the order of integration of the double integral one obtains

$$
\begin{aligned}
\int_{0}^{t} \lambda e^{-\lambda(t-s)} \mathcal{S}(t-s) u(s) d s & =\left(1-e^{-\lambda t}\right) \mathcal{S}(t) u_{0}+\int_{0}^{t}\left(1-e^{-\lambda(t-\xi)}\right) \mathcal{S}(t-\xi) g(\xi) d \xi \\
& =u(t)-e^{-\lambda t} \mathcal{S}(t) u_{0}-\int_{0}^{t} e^{-\lambda(t-s)} \mathcal{S}(t-s) g(s) d s
\end{aligned}
$$


3.2. Existence for the biological equations. To apply these results to the system (3), set $\mathcal{X}=X \times X$, where $X=L^{p}(\Omega)$ for some $1 \leq p<\infty$ or $X=C(\bar{\Omega})$, and let $\mathcal{A}(G, H)=-\left(D_{G} \Delta G, D_{H} \Delta H\right)$ for $G, H \in D(\Delta) \subset X$. Since the Neumann Laplacian generates a contraction analytic semigroup for each choice of the space $X$ given above, $\mathcal{A}$ will likewise generate a contraction analytic semigroup on $\mathcal{X}$. Equations (3) may then be expressed as

$$
\frac{d}{d t}(G, H)+\mathcal{A}(G, H)=-F(G, H)(1,1), \quad(G, H)(0)=\left(G_{0}, H_{0}\right),
$$

where $F: C[0, \infty ; \mathcal{X}) \rightarrow C[0, \infty ; X)$ is given by $F(G, H)(t)=f(G(t), H(t)) u(t)$ with $u$ being the solution of

$$
\frac{d}{d t}\left(\begin{array}{l}
u \\
v
\end{array}\right)+\left[\begin{array}{cc}
a & -b \\
-a & b
\end{array}\right]\left(\begin{array}{l}
u \\
v
\end{array}\right)=\left(\begin{array}{c}
f u \\
0
\end{array}\right), \quad\left(\begin{array}{l}
u \\
v
\end{array}\right)(0)=\left(\begin{array}{l}
u_{0} \\
v_{0}
\end{array}\right),
$$

where it is understood that the coefficients $a, b$, and $f$ are all functions of $(G, H)$. In order to show that $F$ satisfies the Lipschitz hypothesis of Theorem 3.1, we obtain a priori estimates on solutions of the (linear) system of ordinary differential equations (8).

LEMMA 3.4. Let the coefficient $f$ of Eq. (8) be bounded and let the coefficients $a$ and $b$ be nonnegative. Then solutions $(u, v)$ of Eq. (8) satisfy

$$
\|u, v\|_{l^{1}}(t) \leq e^{B t}\left\|u_{0}, v_{0}\right\|_{l^{1}}
$$

where $B$ is the bound on $f$.

Let $(u, v)$ and $(\tilde{u}, \tilde{v})$ be the solutions of Eq. (8) with coefficients $a, b, f$, and $\tilde{a}, \tilde{b}, \tilde{f}$, respectively. Then

$$
\begin{aligned}
& \|u-\tilde{u}, v-\tilde{v}\|_{l^{1}}(t) \\
& \quad \leq e^{B t}\left(\left\|u_{0}-\tilde{u}_{0}, v_{0}-\tilde{v}_{0}\right\|_{l^{1}}+4\left\|\tilde{u}_{0}, \tilde{v}_{0}\right\|_{l^{\infty}} \int_{0}^{t}\|a-\tilde{a}, b-\tilde{b}, f-\tilde{f}\|_{l^{1}}(s) d s\right) .
\end{aligned}
$$

Proof. Let $\left(s_{1}, s_{2}\right)=(\operatorname{sgn}(u), \operatorname{sgn}(v))$ where $\operatorname{sgn}(x)$ is $-1,0$, or 1 if $x$ is negative, zero, or positive respectively. Taking the inner product of $(8)$ with $\left(s_{1}, s_{2}\right)$ gives

$$
\frac{d}{d t}\|u, v\|_{l^{1}}+(a|u|+b|v|)\left(1-s_{1} s_{2}\right)=f|u| \leq B\|u, v\|_{l^{1}}
$$

Since the second term on the left-hand side is nonnegative, discarding it does not alter the validity of the inequality. The $l^{1}$ estimate now follows immediately. We note that while the $\|\cdot\|_{l^{1}}$ norm above may not be differentiable at all times, it is continuous, and the formal arguments can easily be made rigorous.

The difference of the equations for $(u, v)$ and $(\tilde{u}, \tilde{v})$ may be written as

$$
\begin{aligned}
& \frac{d}{d t}\left(\begin{array}{l}
u-\tilde{u} \\
v-\tilde{v}
\end{array}\right)+\left[\begin{array}{cc}
a & -b \\
-a & b
\end{array}\right]\left(\begin{array}{c}
u-\tilde{u} \\
v-\tilde{v}
\end{array}\right) \\
& \quad=f\left(\begin{array}{c}
u-\tilde{u} \\
0
\end{array}\right)+\left[\begin{array}{cc}
a-\tilde{a} & -(b-\tilde{b}) \\
-(a-\tilde{a}) & b-\tilde{b}
\end{array}\right]\left(\begin{array}{l}
\tilde{u} \\
\tilde{v}
\end{array}\right)+(f-\tilde{f})\left(\begin{array}{l}
\tilde{u} \\
0
\end{array}\right) .
\end{aligned}
$$

Taking the inner product with $\left(s_{1}, s_{2}\right)=(\operatorname{sgn}(u-\tilde{u}), \operatorname{sgn}(v-\tilde{v}))$ gives

$$
\frac{d}{d t}\|u-\tilde{u}, v-\tilde{v}\|_{l^{1}} \leq B\|u-\tilde{u}, v-\tilde{v}\|_{l^{1}}+2\|a-\tilde{a}, b-\tilde{b}, f-\tilde{f}\|_{l^{1}}\|\tilde{u}, \tilde{v}\|_{l^{\infty}},
$$


so that

$$
\begin{aligned}
& \|u-\tilde{u}, v-\tilde{v}\|_{l^{1}}(t) \\
& \quad \leq e^{B t}\left\|u_{0}-\tilde{u}_{0}, v_{0}-\tilde{v}_{0}\right\|_{l^{1}}+2 \int_{0}^{t} e^{B(t-s)}\|a-\tilde{a}, b-\tilde{b}, f-\tilde{f}\|_{l^{1}}(s)\|\tilde{u}, \tilde{v}\|_{l^{\infty}}(s) d s .
\end{aligned}
$$

Since $\|\tilde{u}, \tilde{v}\|_{l^{\infty}}(s) \leq\|\tilde{u}, \tilde{v}\|_{l^{1}}(s) \leq \exp (B s)\left\|\tilde{u}_{0}, \tilde{v}_{0}\right\|_{l^{1}}$, and $\|\tilde{u}, \tilde{v}\|_{l^{1}}(0) \leq 2\left\|\tilde{u}_{0}, \tilde{v}_{0}\right\|_{l^{\infty}}$, the result follows.

Corollary 3.5. Let $X$ be $L^{p}(\Omega), 1 \leq p \leq \infty$, or $C(\bar{\Omega})$, and $\mathcal{X}=X \times X$. Then under the hypotheses of the lemma, solutions of Eq. (8) in $\mathcal{X}$ satisfy

$$
\|u, v\|_{\mathcal{X}}(t) \leq C e^{B t}\|u, v\|_{\mathcal{X}}(0)
$$

and

$$
\begin{aligned}
& \|u-\tilde{u}, v-\tilde{v}\|_{\mathcal{X}}(t) \\
& \quad \leq C e^{B t}\left(\left\|u_{0}-\tilde{u}_{0}, v_{0}-\tilde{v}_{0}\right\|_{\mathcal{X}}+C(t)\left\|\tilde{u}_{0}, \tilde{v}_{0}\right\|_{L^{\infty}(\Omega)} \int_{0}^{t}\|a-\tilde{a}, b-\tilde{b}, f-\tilde{f}\|_{\mathcal{X}}(s) d s\right)
\end{aligned}
$$

( $C$ depends only upon $\Omega$ and $C(t)$ depends additionally upon $t$ ).

Corollary 3.6. Let $a, b$, and $f$ mapping $\mathfrak{R}^{2} \rightarrow \mathfrak{R}$ be globally Lipschitz and suppose that $a$ and $b$ are nonnegative and $f$ is bounded. Let $X=L^{p}(\Omega), 1 \leq p \leq \infty$, or $X=C(\bar{\Omega}), \mathcal{X}=X \times X$, and let $u: C[0, \infty, \mathcal{X}) \rightarrow C[0, \infty, X)$ be defined by $(G, H) \mapsto u$ where $u$ is the first component of the solution of (8) with coefficients $a(G, H), b(G, H)$, and $f(G, H)$. If the initial data $u_{0}, v_{0} \in L^{\infty}(\Omega) \cap X$, then for all $t>0$ there exists $K(t)>0$ such that

$$
\|u-\tilde{u}\|_{C[0, t, X]} \leq K(t)\|G-\widetilde{G}, H-\widetilde{H}\|_{C[0, t ; \mathcal{X}]},
$$

where $u=u(G, H)$ and $\tilde{u}=u(\widetilde{G}, \widetilde{H})$.

Theorem 3.7. Let $X=L^{p}(\Omega), 1 \leq p<\infty$, or $X=C(\bar{\Omega})$. Suppose that $a, b: \mathfrak{R}^{2} \rightarrow \mathfrak{R}$ are nonnegative and globally Lipschitz functions and that $f: \mathfrak{R}^{2} \rightarrow \mathfrak{R}$ is bounded and globally Lipschitz. Then for each $G_{0}, H_{0} \in X$ and $u_{0}, v_{0} \in X \cap L^{\infty}(\Omega)$, there exists a unique mild solution $(G, H, u, v) \in C\left[0, \infty ; X^{4}\right)$ of

$$
\begin{gathered}
\frac{d u}{d t}+a(G, H) u-b(G, H) v=f(G, H) u, \\
\frac{d v}{d t}-a(G, H) u+b(G, H) v=0, \\
\frac{\partial G}{\partial t}-D_{G} \Delta G=-f(G, H) u,\left.\quad \frac{\partial G}{\partial n}\right|_{\partial \Omega}=0, \\
\frac{\partial H}{\partial t}-D_{H} \Delta H=-f(G, H) u,\left.\quad \frac{\partial H}{\partial n}\right|_{\partial \Omega}=0,
\end{gathered}
$$


satisfying $(u, v, G, H)(0)=\left(u_{0}, v_{0}, G_{0}, H_{0}\right)$. In this context a mild solution means that $u, v \in C^{1}[0, \infty, X)$ and satisfy the ordinary differential equation in $X$, and

$$
\begin{aligned}
& G(t)=S_{G}(t) G_{0}-\int_{0}^{t} S_{G}(t-s) f(G(s), H(s)) u(s) d s, \\
& H(t)=S_{H}(t) H_{0}-\int_{0}^{t} S_{H}(t-s) f(G(s), H(s)) u(s) d s,
\end{aligned}
$$

where $S_{G}$ and $S_{H}$ are the semigroups generated by $D_{G} \Delta$ and $D_{H} \Delta$ respectively with Neumann boundary conditions.

Suppose additionally that $a(G, H)$ and $b(G, H)$ are bounded on $\Omega \times[0, \delta]$ for some $\delta>0$. Then the mild solution above is a strong solution.

Proof. Letting $\mathcal{X}=X \times X$, existence will follow if the map

$$
F: C[0, t: \mathcal{X}] \rightarrow C[0, t ; X]
$$

defined by $F(G, H)=f(G, H) u$ satisfies the Lipschitz hypothesis of Theorem 3.1. Since $f$ is globally Lipschitz it follows that

$$
\begin{aligned}
\|f(G, H) u-f(\widetilde{G}, \widetilde{H}) \tilde{u}\|_{X} & \leq\|(f(G, H)-f(\widetilde{G}, \widetilde{H})) u\|_{X}+\|f(\widetilde{G}, \widetilde{H})(u-\tilde{u})\|_{X} \\
& \leq M\|G-\widetilde{G}, H-\widetilde{H}\|_{\mathcal{X}}\|u\|_{L^{\infty}(\Omega)}+B\|u-\tilde{u}\|_{X},
\end{aligned}
$$

where $M$ is the Lipschitz constant for $f$ and $B$ is the bound on $f$. Corollary 3.5 and 3.6 now imply

$$
\|F(G, H)-F(\widetilde{G}, \widetilde{H})\|_{C[0, t ; X]} \leq K(t)\|G-\widetilde{G}, H-\widetilde{H}\|_{C[0, t ; \mathcal{X}]} ;
$$

so the hypotheses of Theorem 3.1 are satisfied, implying the existence of a mild solution.

Lemma 3.2 is used to establish that the solutions are strong under the additional hypotheses. For the remainder of the proof, let a tilde over a function indicate the translate of the function in time by $\delta(\tilde{u}(t)=u(t+\delta)$ etc.). The estimates above on $F$ give

$$
\begin{aligned}
\|F(G, H)(t)-F(G, H)(t+\delta)\|_{\mathcal{X}} & \equiv\|F(G, H)-F(\widetilde{G}, \widetilde{H})\|_{\mathcal{X}}(t) \\
& \leq M\|G-\widetilde{G}, H-\widetilde{H}\|_{\mathcal{X}}(t)\|u\|_{L^{\infty}(\Omega)}(t)+B\|u-\tilde{u}\|_{X}(t) .
\end{aligned}
$$

$\|u\|_{L^{\infty}(\Omega)}(t)$ may be bounded by Corollary 3.5 ; so the hypotheses of Lemma 3.2 will be satisfied provided the last term can be bounded appropriately. An application of Corollary 3.5 gives

$$
\begin{aligned}
\|u-\tilde{u}, v-\tilde{v}\|_{\mathcal{X}}(t) & \leq C(t)\left(\left\|u_{0}-u(\delta), v_{0}-v(\delta)\right\|_{\mathcal{X}}+\int_{0}^{t}\|a-\tilde{a}, b-\tilde{b}, f-\tilde{f}\|(s) d s\right) \\
& \leq C(t)\left(\left\|u_{0}-u(\delta), v_{0}-v(\delta)\right\|_{\mathcal{X}}+\int_{0}^{t}\|G-\tilde{G}, H-\tilde{H}\| d s\right) .
\end{aligned}
$$


The hypothesis on the coefficients is now used to estimate the term involving the initial conditions. Integration of the ordinary differential equations for $u$ and $v$ gives

$$
\left(\begin{array}{l}
u \\
v
\end{array}\right)(\tau)-\left(\begin{array}{l}
u_{0} \\
v_{0}
\end{array}\right)=\int_{0}^{\tau}\left[\left(\begin{array}{c}
f u \\
0
\end{array}\right)-\left[\begin{array}{cc}
a & -b \\
-a & b
\end{array}\right]\left(\begin{array}{l}
u \\
v
\end{array}\right)\right], \quad 0 \leq \tau \leq \delta
$$

so that

$$
\begin{aligned}
\left\|u_{0}-u(\tau), v_{0}-v(\tau)\right\|_{\mathcal{X}} & \leq B \int_{0}^{\tau}\|u, v\|_{\mathcal{X}} \\
& \leq B\left(\tau\left\|u_{0}, v_{0}\right\|_{\mathcal{X}}+\int_{0}^{\tau}\left\|u_{0}-u, v_{0}-v\right\|\right) .
\end{aligned}
$$

An application of Gronwall's inequality now establishes that

$$
\left\|u_{0}-u(\delta), v_{0}-v(\delta)\right\|_{\mathcal{X}} \leq C(T) \delta .
$$

Combining the above estimates shows that the nonlinear term $F$ satisfies the hypotheses of Lemma 3.2, guaranteeing a strong solution.

REMARK. Even if $a$ and $b$ are not globally bounded, bounds on $a(G, H)$ and $b(G, H)$ still follow when $X=C(\bar{\Omega})$, since then $(G, H) \in C[0, \infty ; \mathcal{X})$, and $a$ and $b: \mathfrak{R}^{2} \rightarrow \mathfrak{R}$ continuous implies $a(G, H)$ and $b(G, H)$ are continuous, and hence bounded on $\bar{\Omega} \times[0, T]$.

The coefficient $f$ for the biological equations (3) is frequently chosen according to Monod's law [17, 19], i.e.,

$$
f(G, H)=V_{0} \frac{G^{+} H^{+}}{K_{0}+G^{+} H^{+}},
$$

where a plus superscript indicates the positive part ( $a^{+}=a$ if $a \geq 0$ and zero otherwise). This function is not globally Lipschitz on $\mathfrak{R}^{2}$. While $\xi \mapsto \xi /(1+\xi)$ is globally Lipschitz, multiplication is not, so the product $G^{+} H^{+}$causes a problem. However, the maximum principle can be used to circumvent this technicality.

Corollary 3.8. Assume that the hypotheses of the above theorem hold except that $f$ is given as above. Suppose additionally that the initial data for $G_{0}$ and $H_{0}$ are bounded above, and that the initial data $u_{0}, v_{0}$ are nonnegative. Then the conclusions of the theorem remain valid. Moreover, $u$ and $v$ are nonnegative for all time, and $\max _{\Omega} G(t) \leq$ $\max _{\Omega} G_{0}, \max _{\Omega} H(t) \leq \max _{\Omega} H_{0}$.

Proof. We begin by observing that if $B=\max \left(\max _{\Omega} G_{0}, \max _{\Omega} H_{0}\right)$ then the function

$$
\hat{f}(G, H)=V_{0} \frac{\min \left(G^{+}, B\right) \min \left(H^{+}, B\right)}{K_{0}+\min \left(G^{+}, B\right) \min \left(H^{+}, B\right)}
$$

is globally Lipschitz, so that we may consider the corresponding solution $(u, v, G, H)$ guaranteed by the theorem.

Observe that the solutions of

$$
\begin{array}{ll}
\frac{d \tilde{u}}{d t}+a \tilde{u}=f|\tilde{u}|+b|\tilde{v}|, & u(0)=u_{0} \\
\frac{d \tilde{v}}{d t}+b \tilde{v}=a|\tilde{u}|, & v(0)=v_{0}
\end{array}
$$


are nonnegative when the initial data is nonnegative. In this situation we can omit the absolute values from the right-hand sides to obtain solutions of (8). By uniqueness, it follows that $u=\tilde{u}$ and $v=\tilde{v}$ are nonnegative.

Next recall that the heat semigroup with Neumann boundary conditions maps nonnegative functions to nonnegative functions. Since $\hat{f}(G, H)$ and $u$ are nonnegative, the integral expressions

$$
\begin{aligned}
& G(t)=S_{G}(t) G_{0}-\int_{0}^{t} S_{G}(t-s)(\hat{f} u)(s) d s, \\
& H(t)=S_{H}(t) G_{0}-\int_{0}^{t} S_{H}(t-s)(\hat{f} u)(s) d s
\end{aligned}
$$

show that $G(t) \leq S_{G}(t) G_{0}$ and $H(t) \leq S_{H}(t) H_{0}$ pointwise. But $S_{G}(\cdot) G_{0}$ and $S_{H}(\cdot) H_{0}$ are just solutions of the homogeneous heat equation. Thus $\max _{\Omega} G(t) \leq \max _{\Omega} G_{0} \leq B$ and $\max _{\Omega} H(t) \leq \max _{\Omega} H_{0} \leq B$. It follows that $\hat{f}(G, H)=f(G, H)$, and $(u, v, G, H)$ is a solution of the original problem.

4. Long-time behavior. The bounds obtained for $u$ and $v$ all grew exponentially with time. If Eqs. (3) accurately model the biological experiments discussed in the Introduction, we expect the solution to approach a steady state at large times. We show that under reasonable hypotheses on the coefficients $a, b$, and $f$ that this is indeed the case.

H1. The coefficients $a$ and $b$ are nonnegative bounded functions from $\mathfrak{R}^{2}$ to $\mathfrak{R}$.

H2. The initial data $u_{0}, v_{0}, G_{0}$, and $H_{0}$ are all nonnegative and bounded.

H3. The function $f: \mathfrak{R}^{2} \rightarrow \mathfrak{R}$ is of the form

$$
f(G, H)=V_{0} \frac{G^{+} H^{+}}{K_{0}+G^{+} H^{+}}
$$

where $V_{0} \geq 0$ and $K_{0}>0$ are constants.

H4. $b(\cdot, \cdot)$ vanishes at points where $a(\cdot, \cdot)>0$ and $f(\cdot, \cdot)>0$ at points where $a(\cdot, \cdot)=0$. The following properties of the Neumann heat equation will be used below. We denote by $S(\cdot)$ the semigroup generated by the Neumann Laplacian on $L^{p}(\Omega), 1 \leq p<\infty$, or $X=C(\bar{\Omega})$.

- For each $u \in X$ and $t \geq 0, \int_{\Omega} S(t) u=\int_{\Omega} u$.

- If $u \geq 0, t \geq 0$ then $S(t) u \geq 0$.

- For any $u \in X, \lim _{t \rightarrow \infty} S(t) u=\bar{u}$ in $X$, where $\bar{u}$ is the constant function having the same average as $u$.

Lemma 4.1. Let hypotheses H1-H3 hold. Then the mild solutions given by Corollary 3.8 satisfy

- each component is nonnegative,

- $\|G(t)\|_{L^{\infty}(\Omega)} \leq\left\|G_{0}\right\|_{L^{\infty}(\Omega)}$ and $\|H(t)\|_{L^{\infty}(\Omega)} \leq\left\|H_{0}\right\|_{L^{\infty}(\Omega)}$,

- the following conservation property holds:

$$
\int_{\Omega}(G+U)(t)=\int_{\Omega}(G+U)(0), \quad \int_{\Omega}(H+U)(t)=\int_{\Omega}(H+U)(0),
$$

where $U=u+v$. 
Proof. Recall that $u$ and $v$ are nonnegative, and that $G$ and $H$ are bounded above. The $L^{\infty}(\Omega)$ estimates on $G$ and $H$ will then follow if we show that they are nonnegative.

We show that $G$ is nonnegative, the proof for $H$ being identical. Define $\psi:[0, \infty) \rightarrow$ $L^{\infty}(\Omega)$ by

$$
\psi= \begin{cases}V_{0} \frac{H^{+}}{K_{0}+G^{+} H^{+}} u, & G>0 \\ 0 & \text { otherwise }\end{cases}
$$

so that $G$ is the unique solution of

$$
G(t)=S_{G}(t) G_{0}-\int_{0}^{T} S_{G}(t-s) \psi(s) G(s) d s .
$$

Note that $\psi(\cdot) \geq 0$ since $u \geq 0$. Corollary 3.5 shows that for $0 \leq t \leq T,\|u(t)\|_{L^{\infty}(\Omega)} \leq$ $C(T)\left\|u_{0}\right\|_{L^{\infty}(\Omega)}$, and since $H$ is bounded above it follows that $\|\psi(t)\|_{L^{\infty}(\Omega)} \leq C(T)$.

An application of Lemma 3.3 shows that for any $\lambda \geq 0$

$$
G(t)=e^{-\lambda t} S_{G}(t) G_{0}+\int_{0}^{t} e^{-\lambda(t-s)} S_{G}(t-s)[\lambda-\psi(s)] G(s) d s
$$

in particular, for $T>0$ given, $\lambda$ may be chosen so that $\lambda-\psi(s) \geq 0$ for $0 \leq s \leq$ $T$. We claim that solutions of this integral equation must be nonnegative when $G_{0}$ is nonnegative. To see this, consider solutions of the integral equation

$$
\widetilde{G}(t)=e^{-\lambda t} S_{G}(t) G_{0}+\int_{0}^{t} e^{-\lambda(t-s)} S_{G}(t-s)[\lambda-\psi(s)]|\widetilde{G}(s)| d s
$$

(this has a unique solution by Banach's fixed point theorem [3]). Since $S_{G}(\cdot)$ carries nonnegative functions to nonnegative functions, it is clear that $\widetilde{G}(\cdot)$ is nonnegative. But then we may omit the absolute value from the right-hand term, giving a solution of the integral equation for $G$. By uniqueness $G(\cdot)=\widetilde{G}(\cdot) \geq 0$.

Finally, we establish the conservation property. Adding the equations for $u$ and $v$ and integrating gives

$$
\frac{d}{d t} \int_{\Omega} U=\int_{\Omega} f(G, H) u
$$

Integrating the expression for $G$, and recalling that the heat semigroup with Neumann boundary conditions preserves averages, gives

$$
\int_{\Omega} G(t)=\int_{\Omega} G_{0}-\int_{0}^{t} \int_{\Omega} f(G, H) u d s,
$$

so that

$$
\int_{\Omega} G(t)=\int_{\Omega} G_{0}-\int_{0}^{t} \frac{d}{d s} U(s) d s=\int_{\Omega} G_{0}-\int_{\Omega}[U(t)-U(0)] .
$$

We can now establish the existence of a steady state limit as $t \rightarrow \infty$.

THEOREM 4.2. Let hypotheses $\mathrm{H} 1-\mathrm{H} 3$ hold and let $(u, v, G, H)$ be the solution of the biological equations guaranteed by Corollary 3.8. Then 
- $f(G, H) u \in L^{1}\left(0, \infty ; L^{1}(\Omega)\right)$, i.e.,

$$
\int_{0}^{\infty}\left(\int_{\Omega}(f u)(s)\right) d s<\infty
$$

(recall that $f u \geq 0$ ).

- $\lim _{t \rightarrow \infty} G(t)=\bar{G}$ and $\lim _{t \rightarrow \infty} H(t)=\bar{H}$, in $L^{1}(\Omega)$, where $\bar{G}$ and $\bar{H}$ are constant functions.

- If additionally $\mathrm{H} 4$ holds, then $\lim _{t \rightarrow \infty} u(t) \rightarrow 0$ in $L^{1}(\Omega)$, and $\lim _{t \rightarrow \infty} v(t)$ exists in $L^{1}(\Omega)$.

Proof. Adding the equations for $u$ and $v$ gives $d U / d t=f u$, where $U=u+v$. Integrating this expression over space and time gives

$$
\int_{0}^{t} \int_{\Omega} f u=\int_{\Omega} U(t)-U_{0} \leq \int_{\Omega} U(t)+G(t)-U_{0}=\int_{\Omega} G_{0}
$$

(we used the fact that $G$ is nonnegative). Since $f$ and $u$ are nonnegative, it follows that $f u \in L^{1}\left(0, \infty ; L^{1}(\Omega)\right)$.

We show that $G(t) \rightarrow \bar{G}$, the proof for $H(\cdot)$ being identical. Fix $\varepsilon>0$, and let $s$ be chosen so large that $\int_{s}^{\infty} \int_{\Omega}(f u)<\varepsilon$. For $t \geq s$ write

$$
G(t)=S(t) G_{0}-S_{G}(t-s) \int_{0}^{s} S_{G}(s-\xi)(f u)(\xi) d \xi-\int_{s}^{t} S_{G}(t-\xi)(f u)(\xi) d \xi .
$$

Note that the first two terms on the right converge to some constant $C_{\varepsilon}$ in $L^{1}(\Omega)$ as $t \rightarrow \infty$. Since $S_{G}(\cdot)$ is a contraction semigroup, it follows that

$$
\limsup _{t \rightarrow \infty}\left\|G(t)-C_{\varepsilon}\right\|_{L^{1}(\Omega)} \leq \int_{s}^{\infty} \int_{\Omega}(f u) \leq \varepsilon .
$$

Then

$$
\left\|G\left(t_{1}\right)-G\left(t_{2}\right)\right\|_{L^{1}(\Omega)} \leq 2 \varepsilon, \quad t_{1}, t_{2} \geq s,
$$

i.e., $\{G(t)\}_{t \geq 0}$ is Cauchy in $L^{1}(\Omega)$, so converges to a limit $\bar{G} \in L^{1}(\Omega)$. We claim that $\bar{G}$ is constant. Indeed,

$$
|\Omega|\left|C_{\varepsilon}\right|=\left\|C_{\varepsilon}-\bar{G}+\bar{G}\right\|_{L^{1}(\Omega)} \leq \varepsilon+\|\bar{G}\|_{L^{1}(\Omega)}
$$

implies that the sequence $\left\{C_{\varepsilon}\right\}_{\varepsilon>0}$ is bounded; so we may pass to a subsequence $C_{\varepsilon} \rightarrow C$. Then $\left\|\bar{G}-C_{\varepsilon}\right\|_{L^{1}(\Omega)} \leq \varepsilon$; so letting $\varepsilon \rightarrow 0$ gives $\|\bar{G}-C\|_{L^{1}(\Omega)}=0$, i.e., $\bar{G}=C$.

We next show that $u(t) \rightarrow 0$. The key point is to establish $L^{\infty}$ bounds on $u(\cdot)$. Recall that the $L^{\infty}(\Omega)$ bounds of Corollary 3.5 all grew exponentially in time. Below we show that we can get $L^{\infty}$ bounds for $u$ on arbitrarily large subsets of $\Omega$.

Since $a(t)=a(G(t), H(t)), b(t)=b(G(t), H(t))$, and $f(t)=f(G(t), H(t))$ are all bounded, it follows from the dominated convergence theorem that $a(t) \rightarrow a_{\infty}, b(t) \rightarrow b_{\infty}$, and $f(t) \rightarrow f_{\infty}$ in $L^{1}(\Omega)$ as $t \rightarrow \infty$, where $a_{\infty}=a(\bar{G}, \bar{H})$ etc. are all nonnegative constants. Note that hypothesis $\mathrm{H} 4$ shows that either $a_{\infty}>0$ and $b_{\infty}=0$, or $0=a_{\infty}<$ $f_{\infty}$.

Adding the equations for $u$ and $v$ gives $d U / d t=d(u+v) / d t=f u \geq 0$, so that pointwise $U(t)$ is monotone increasing. Lemma 4.1 shows that $\|U(t)\|_{L^{1}(\Omega)}$ is bounded; 
so by the monotone convergence theorem $U \rightarrow U_{\infty}$ in $L^{1}(\Omega)$. Given any $\varepsilon>0$ there is a $\delta>0$ such that

$$
|A| \leq \delta \Rightarrow \int_{A} U_{\infty} \leq \varepsilon
$$

and since $0 \leq u(t) \leq U(t) \leq U_{\infty}$ (pointwise) it follows that

$$
|A| \leq \delta \Rightarrow \int_{\Omega} u \leq \int_{\Omega \backslash A} u+\varepsilon .
$$

Also, there exists $M=M_{\varepsilon}>0$ such that $|\Omega| \leq|E|+\varepsilon$, where $E=E_{\varepsilon}=\{x \in$ $\left.\Omega \mid U_{\infty}(x) \leq M\right\}$. Then

$$
\|u(t)\|_{L^{\infty}(E)} \leq M \quad \text { and } \quad\|v(t)\|_{L^{\infty}(E)} \leq M .
$$

We now consider the two cases $a_{\infty}>0, b_{\infty}=0$ and $0=a_{\infty}<f_{\infty}$ separately.

If $a_{\infty}>0$ and $b_{\infty}=0$, the equation for $u$ gives

$$
\frac{d}{d t} \int_{E} u+a_{\infty} \int_{E} u=\int_{E} f u+\int_{E}\left(a_{\infty}-a\right) u+b v .
$$

Integrating from $s$ to $t$ and using the $L^{\infty}$ bounds of $u$ and $v$ on $E$ gives

$$
\begin{aligned}
\int_{E} u(t) \leq e^{-a_{\infty}(t-s)} & \int_{E} u(s) \\
& +\int_{s}^{t} e^{-a_{\infty}(t-\xi)}\left(M\left\|a_{\infty}-a\right\|_{L^{1}(\Omega)}+M\|b\|_{L^{1}(\Omega)}+\|f u\|_{L^{1}(\Omega)}\right)(\xi) d \xi .
\end{aligned}
$$

Since $\left\|a_{\infty}-a\right\|_{L^{1}(\Omega)}$ and $\|b\|_{L^{1}(\Omega)} \rightarrow 0$, and $\int_{s}^{\infty}\|f u\|_{L^{1}(\Omega)} \rightarrow 0$, there exists $s_{0}>0$ such that

$$
\left\|a_{\infty}-a\right\|_{L^{1}(\Omega)}(s)+\|b\|_{L^{1}(\Omega)}(s) \leq \frac{\varepsilon}{M a_{\infty}}
$$

and

$$
\int_{s}^{\infty}\|f u\|_{L^{1}(\Omega)} \leq \varepsilon, \quad s>s_{0}
$$

Then for $t \geq s_{0}$,

$$
\int_{E} u(t) \leq e^{-a_{\infty}\left(t-s_{0}\right)} \int_{E} u\left(s_{0}\right)+2 \varepsilon \rightarrow 2 \varepsilon
$$

Since

$$
\int_{\Omega} u(t) \leq \int_{E} u(t)+\varepsilon
$$

it follows that $u(t) \rightarrow 0$ as $t \rightarrow \infty$.

We now consider the situation $0=a_{\infty}<f_{\infty}$. Again we consider the equation for $u$ :

$$
\begin{aligned}
\frac{d u}{d t}-f_{\infty} u & =\left[\left(f-f_{\infty}\right)-a\right] u+b v \\
& \geq-\left|\left(f-f_{\infty}\right)-a\right| M
\end{aligned}
$$

so that

$$
\int_{E} u(t) \geq e^{f_{\infty}(t-s)} \int_{E} u(s)-M \int_{s}^{t} e^{f_{\infty}(t-\xi)}\left\|\left(f-f_{\infty}\right)-a\right\|_{L^{1}(\Omega)}(\xi) d \xi
$$


If $\int_{E} u(t) \nrightarrow 0$, then there is a sequence of times $\left\{s_{n}\right\}_{n=0}^{\infty}$ and $\delta>0$ such that $s_{n} \rightarrow \infty$, and $\int_{E} u\left(s_{n}\right) \geq \delta$. Select $s_{n}$ so large that

$$
\left\|\left(f_{\infty}-f\right)-a\right\|_{L^{1}(\Omega)} \leq \frac{\delta}{2 f_{\infty} M}
$$

to get

$$
\int_{E} u(t) \geq \frac{\delta}{2} e^{f_{\infty}\left(t-s_{n}\right)} \rightarrow \infty \quad \text { as } t \rightarrow \infty .
$$

This contradicts $\int_{E} u \leq \int_{\Omega} u \leq \int_{\Omega} U_{\infty}<\infty$.

Since $v=U-u$, it follows that $v(t) \rightarrow U_{\infty}$ as $t \rightarrow \infty$ in $L^{1}(\Omega)$.

5. Summary. Analysis of a hysteretic reaction-diffusion system is presented in this paper. Our results suggest that hysteretic reaction-diffusion systems may provide a model for accretion pattern formation in cell biology.

Different torpor levels were used to identify different states of cell growth in response to moving gradients of substrate. An important assumption, based on biological observations, is that the transition matrix simulating cell switching between torpor levels has a hysteretic dependence on availability of substrate. Due to the hysteresis and nonlinearity of chemical reaction functions, various kinds of patterns are observed and they resemble the results from biological experiments.

Existence, uniqueness, and long-time behavior of the solution are established for a hysteretic reaction-diffusion system with two torpor levels and two substrate concentrations. Currently, numerical schemes to approximate solutions of the hysteretic reaction-diffusion systems are being developed and analyzed.

\section{REFERENCES}

[1] N. E. Britton, Reaction-Diffusion Equations and Their Applications to Biology, Academic Press, New York, 1986

[2] E. O. Budriené, A. A. Polezhaev, and M. O. Ptitsyn, Mathematical modeling of intercellular regulation causing the formation of spatial structures in bacterial colonies, J. Theor. Biol. 135, 323-341 (1988)

[3] J. A. Goldstein, Semigroups of Linear Operators and Applications, Oxford Mathematical Monographs, The Clarendon Press, Oxford University Press, New York, 1985

[4] C. Chiu, F. C. Hoppensteadt, and W. Jäger, Analysis and computer simulation of accretion patterns in bacterial cultures, to appear

[5] C. Chiu and N. J. Walkington, An ADI method for hysteretic reaction-diffusion systems, SIAM J. Numer. Anal. 34, 1185-1206 (1997)

[6] F. C. Hoppensteadt and W. Jäger, Pattern formation by bacteria, Lecture Notes in Biomath. 38, 68-81 (1980)

[7] F. C. Hoppensteadt, W. Jäger, and C. Pöppe, A hysteresis model for bacterial growth patterns, in Modelling of Patterns in Space and Time, W. Jäger and J. D. Murray, eds., Lecture Notes in Biomath. vol. 55, Springer-Verlag, Berlin, New York, 1984, pp. 123-134

[8] G. Hauser, Uber Fäulnisslacterien und deren Beriehungen zur Septicämie, Leipzig: F. G. W. Vogel, 1885

[9] U. Hornung and R. E. Showalter, Pde-models with hysteresis on the boundary, in A. Visintin, editor, Models of Hysteresis, volume 286, Research Notes in Mathematics, Pitman, 1983, pp. 30-38

[10] U. Hornung and R. E. Showalter, Elliptic-parabolic equations with hysteresis boundary conditions, SIAM J. Mathematical Analysis 26, 775-790 (1995)

[11] D. L. Lewis and D. K. Gattie, The ecology of quiescent microbes, ASM News 57, 27-32 (1991) 
[12] P. Gerhardt, et al., Manual of Methods for General Bacteriology, P. Gerhardt, editor-in-chief, Amer. Soc. for Microbiology, Washington, DC 20006, 1981

[13] H. M. Lieberstein, Theory of Partial Differential Equations, Mathematics in Science and Engineering, vol. 93, Academic Press, New York and London, 1972

[14] T. D. Little and R. E. Showalter, Semilinear parabolic equations with Preisach hysteresis, Differential and Integral Equations 7, 1021-1040 (1994)

[15] J. W. T. Wimpenney, J. P. Coombs, R. W. Lovitt, and S. G. Whittaker, A gel-stabilized model ecosystem for investigating microbial growth in spatially ordered solute gradients, J. Gen. Microbio. 127, 277-287 (1981)

[16] J. W. Macki, P. Nistri, and P. Zecca, Mathematical Models for Hysteresis, SIAM Review, Vol. 35, No. 1, 1993, pp. 94-123

[17] J. Monod, Recherches sur La Croissance de Cultures Bacteriennes, Hermann et Cie, Paris, 1942

[18] J. D. Murray, Mathematical Biology, Biomathematics Texts, Springer-Verlag, 1989

[19] T. E. Shehata and A. G. Marr, Synchronous growth of enteric bacteria, J. Bacteriology 103, 789 (1970) 